\title{
Variable anisotropy of small-scale stratospheric irregularities retrieved from stellar scintillation measurements by GOMOS/Envisat
}

\author{
V. Kan ${ }^{1}$, V. F. Sofieva ${ }^{2}$, and F. Dalaudier ${ }^{3}$ \\ ${ }^{1}$ A.M. Obukhov Institute of Atmospheric Physics Russian Academy of Sciences, Moscow, Russia \\ ${ }^{2}$ Finnish Meteorological Institute, Helsinki, Finland \\ ${ }^{3}$ Université Versailles St-Quentin, UPMC University Paris 06, CNRS/INSU, LATMOS-IPSL, 78280 Guyancourt, France \\ Correspondence to: V. F. Sofieva (viktoria.sofieva@fmi.fi)
}

Received: 27 December 2013 - Published in Atmos. Meas. Tech. Discuss.: 10 February 2014

Revised: 14 May 2014 - Accepted: 16 May 2014 - Published: 25 June 2014

\begin{abstract}
In this paper, we consider possibilities for studying the anisotropy of small-scale air density irregularities using satellite observations of bi-chromatic stellar scintillations during tangential occultations. Estimation of the anisotropy coefficient (the ratio of the characteristic horizontal to vertical scales) and other atmospheric parameters is based on the comparison of simulated/theoretical and experimental autospectra and coherency spectra of scintillation. Our analyses exploit a 3-D model of the spectrum of atmospheric inhomogeneities, which consists of anisotropic and isotropic components. For the anisotropic component, a spectral model with variable anisotropy is used. Using stellar scintillation measurements by GOMOS (Global Ozone Monitoring by Occultation of Stars) fast photometers, estimates of the anisotropy coefficient are obtained for atmospheric irregularities with vertical scales of 8-55 m at altitudes of 43-30 km. It is shown that the anisotropy increases from about 10 to 50 with increasing vertical scales.
\end{abstract}

\section{Introduction}

Random irregularities of air density and temperature, which are generated by internal gravity waves (GWs) and turbulence, cause fluctuations in light intensity (scintillation) when a star is observed through the atmosphere. Stellar scintillations in occultation experiments are widely used for probing the atmospheric irregularities of planets and their satellites (Hubbard et al., 1988; Raynaud et al., 2004) and the Earth atmosphere (Gurvich and Kan, 2003a, b; Gurvich et al., 2007; Sofieva et al., 2007a, b, 2009). The phase screen approximation (Gurvich, 1984; Hubbard et al., 1978) and the theory of weak scintillations (Tatarskii, 1961, 1971) provide the basis for analyses of scintillation measurements and reconstructing the parameters of atmospheric irregularities.

Stellar scintillation measurements on board the orbital station MIR (Alexandrov et al., 1990; Gurvich and Kan, 2003a, b) have shown that there are two types of irregularities in the stratosphere: relatively large anisotropic irregularities and small-scale isotropic ones. Based on these data, an empirical model of the 3-D wave-number spectrum of air density irregularities has been developed. In this spectrum, the model of saturated gravity waves is used for description of the anisotropic component, and the Kolmogorov model is used for the description of the isotropic component (Gurvich and Brekhovskikh, 2001; Gurvich and Kan, 2003b). The methodology for reconstructing the parameters of the 3-D model of atmospheric irregularities from scintillation measurements has been successfully applied for analyses of observations on board the MIR station (Gurvich and Kan, 2003a, b), and then for mass reconstruction of the atmospheric irregularity parameters from measurements by the GOMOS fast photometers on board the Envisat satellite (Sofieva et al., 2007a, 2009). This method allows reconstructing all parameters of the 3-D spectral model, except for the anisotropy coefficient $\eta$ of the anisotropic component ( $\eta$ is defined as a ratio of characteristic horizontal to vertical scale) and therefore $\eta=$ Const $\gg 1$ was assumed in retrievals. A detailed analysis 
of the methods and results of the stellar scintillation observations can be found in the review by Sofieva et al. (2013).

Analyses of satellite measurements by CRISTA (Ern et al., 2004, 2011) and HIRDLS (Alexander et al., 2008), radiosonde data (Wang et al., 2005) and GPS radio occultation measurements by COSMIC (Wang and Alexander, 2010) all indicate strong anisotropy of large-scale (dominant) irregularities in the stratospheric gravity wave spectrum with estimates of $\eta$ being of the order of one to several hundreds. The anisotropy of the small-scale irregularities were much less investigated. Of the known data, we note the estimate $\eta \approx 15-$ 20 for the vertical scales $\sim 10-20$ m obtained from the scintillation measurements of a double star by GOMOS (Kan et al., 2012). The estimate $\eta \approx 10$ for the vertical scale of $3.2 \mathrm{~m}$ has been obtained from angular dependence of backscattered radar signals (Gurvich and Kon, 1993). (It is interesting to note that atmospheric radars are directly sensitive to the 3D spectra of irregularities, and consequently, the retrieval is much less dependent on the assumed model, but radars are sensitive to a single scale equal to half their wavelength.) Although small-scale irregularities are also characterized by significant anisotropy, it is much smaller than for largescale irregularities. The concept of 3-D spectrum of atmospheric inhomogeneities with variable anisotropy decreasing toward small scales has been discussed in Dalaudier and Gurvich (1997), Gurvich and Chunchuzov (2008a, b) and Gurvich (1997). The model of GW spectrum with variable anisotropy (Gurvich and Chunchuzov, 2008a, b) described satisfactorily not only the experimental 1 -D vertical wavenumber spectrum but also the horizontal spectra having domains with different slopes.

Depending on the angular distance of the star from the satellite orbital pole, various sensing trajectories in the atmosphere can be realized: from vertical occultations (the star is in the orbital plane) to tangential occultations (the star is near the orbital pole, i.e., near the "North" pole with respect to the satellite rotation); this is illustrated in the left part of Fig. 1 in Dalaudier et al. (2001). During tangential occultations, the star descends down to some minimal height $h_{0}$, and then rises back. Around $h_{0}$, the trajectory of the ray perigee is practically horizontal. Scintillations are mainly sensitive to small-scale irregularities (Tatarskii, 1971), therefore the observations of stellar scintillations are primarily aimed at the study of small-scale structures.

Availability of two fast photometers in the GOMOS mission with sampling rate of $1 \mathrm{kHz}$ operating at blue (473$\left.527 \mathrm{~nm}, \lambda_{\mathrm{B}} \approx 500 \mathrm{~nm}\right)$ and red $\left(646-698 \mathrm{~nm}, \lambda_{\mathrm{R}} \approx 672 \mathrm{~nm}\right)$ wavelengths allows using the properties of cross- and coherency spectra of chromatic scintillations, in addition to scintillation auto-spectra. Due to the dispersion of regular refraction, rays of different colors have different trajectories in the atmosphere, which are shifted vertically by a distance $\Delta_{c}$ (chromatic shift corresponding to the ray perigee) (Dalaudier et al., 2001). The properties of chromatic scintillations depend on the type of atmospheric irregularities, occultation geometry and $\Delta_{c}$ (Gurvich et al., 2005; Kan, 2004; Kan et al., 2001).

The main objective of the present paper is to estimate the anisotropy coefficient of small-scale irregularities by measuring the horizontal spectra of chromatic scintillations of stars during tangential occultation by GOMOS/ENVISAT. The horizontal coherency scale of the anisotropic component of scintillations depends on the vertical scale $2 \pi \Delta_{c}$ of atmospheric irregularities and the corresponding anisotropy coefficient. Estimates of the anisotropy coefficient are obtained for irregularities with vertical scales from 8 to $55 \mathrm{~m}$ for ray perigee altitudes 43 to $30 \mathrm{~km}$. The investigated range of inhomogeneity scales is close to the vertical scale of gravity wave breaking (Gurvich and Chunchuzov, 2003; Gurvich and Kan, $2003 a, b)$, where one can expect significant changes in the anisotropy of irregularities.

The paper is organized as follows. Section 2 discusses the basic assumptions and approximations used in the analysis. In Sect. 3, we consider the geometry of tangential occultations, the properties of chromatic scintillations and discuss their utilization for estimating the parameters of atmospheric inhomogeneities. Section 4 presents the measurement results and comparisons with theoretical calculations. Discussion and summary conclude the paper (Sect. 5).

\section{Main approximations}

In our analysis, we used a model of a two-component 3-D spectrum of atmospheric inhomogeneities, the phase screen approximation and the assumption of weak scintillation.

\subsection{A model of the 3-D spectrum of atmospheric irregularities}

We describe the random field of relative fluctuations of refractivity (or air density) by its 3-D wave-number spectrum $\Phi_{v}(\boldsymbol{\kappa}), \boldsymbol{\kappa}$ being wave number. We assume that $\Phi_{v}$ is the sum of two statistically independent components corresponding to the anisotropic and isotropic irregularities, $\Phi_{\mathrm{W}}(\kappa)$ and $\Phi_{K}(\kappa)$, respectively (Gurvich and Brekhovskikh, 2001):

$\Phi_{\nu}(\kappa)=\Phi_{\mathrm{W}}(\kappa)+\Phi_{K}(\kappa)$.

In previous works, a phenomenological 3-D model with constant anisotropy was used for characterization of the anisotropic component $\Phi_{\mathrm{W}}$ (Gurvich and Brekhovskikh, 2001; Gurvich and Kan, 2003a, b; Sofieva et al., 2007a, b, 2009). Since several studies mentioned above indicate a variable anisotropy growing with the scale of irregularities (the results of this work also support this, see below) we will use for $\Phi_{\mathrm{W}}$ the model with variable anisotropy, which depends on the vertical scale (Gurvich and Chunchuzov, 2008a, b). This model is a generalization of the 3-D spectrum of gravity waves with constant anisotropy for irregularities larger than the GW breaking scale (Chunchuzov, 2002). We present the 

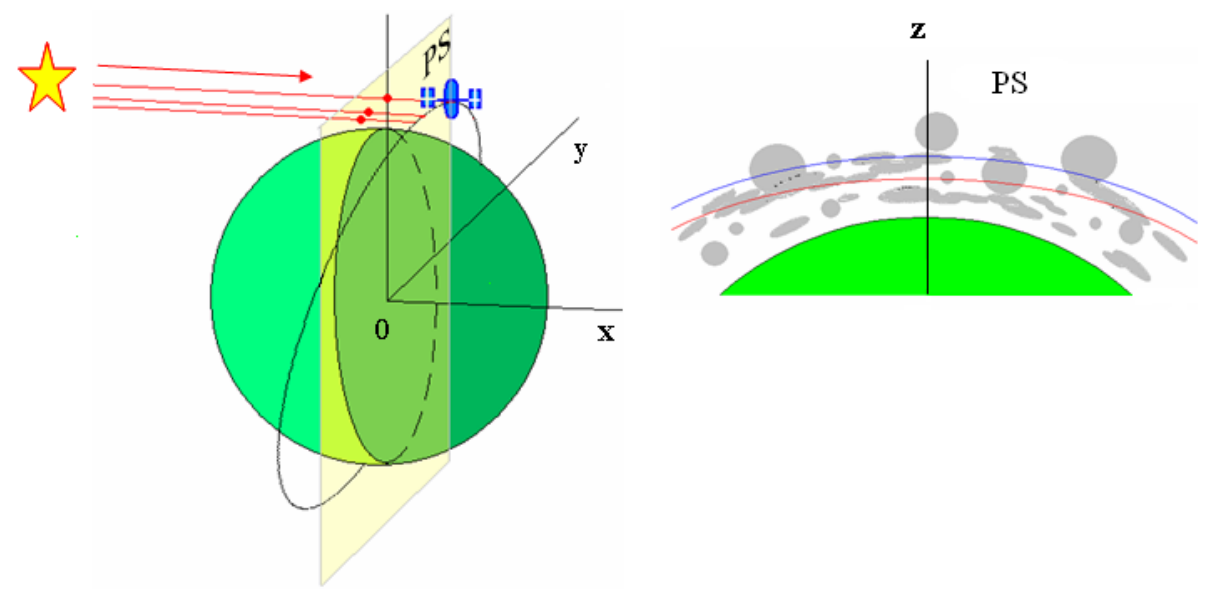

Figure 1. Left: the scheme of red rays observations: the phase screen plane (PS, highlighted in yellow) is in the plane $y 0 z$, which is perpendicular to incident rays. Right: trajectories of rays in the phase screen: the trajectories of red and blue rays are separated vertically by the chromatic shift $\Delta_{c}$. Atmospheric irregularities are schematically shown by gray circles and ellipses.

model of $\Phi_{\mathrm{W}}(\kappa)$ (Gurvich and Chunchuzov, 2008a, b) as follows:

$$
\begin{aligned}
\Phi_{\mathrm{W}}\left(\kappa_{z}, \kappa_{\perp}\right)= & \frac{1}{2} C_{\mathrm{W}} \eta^{2}\left(\kappa_{z}\right)\left(\kappa_{z}^{2}+K_{z, \mathrm{~W}}^{2}\right)^{-5 / 2} \\
& \exp \left[-\frac{\kappa_{\perp}^{2} \eta^{2}\left(\kappa_{z}\right)}{\kappa_{z}^{2}}\right] \phi\left(\kappa_{z} / \kappa_{z, \mathrm{~W}}\right),
\end{aligned}
$$

where $\kappa_{z}$ is the vertical wave number, $\kappa_{\perp}=\sqrt{\kappa_{x}^{2}+\kappa_{y}^{2}}, \kappa_{x}, \kappa_{y}$ are horizontal wave numbers, $C_{\mathrm{W}}$ is the structure characteristic, $K_{z, \mathrm{~W}}$ and $\kappa_{z, \mathrm{~W}}$ are wave numbers corresponding to outer $L_{\mathrm{W}}=2 \pi / \mathrm{K}_{z, \mathrm{~W}}$ and inner $l_{\mathrm{W}}=2 \pi / \kappa_{z, \mathrm{~W}}$ vertical scales, respectively, and the function $\phi\left(\kappa_{z} / \kappa_{z, \mathrm{~W}}\right)$ defines the decay of the spectrum at small scales $\left|\kappa_{z}\right| \geq \kappa_{z}$,W. We use the decay function in the form: $\phi=\left(1+\kappa_{z}^{2} / \kappa_{z, \mathrm{~W}}^{2}\right)^{-1}$ (Gurvich and Kan, 2003a, b; Sofieva et al., 2007a).

The 3-D spectrum (Eq. 2) has axial symmetry with respect to the $\kappa_{z}$ axis. The function $\eta\left(\kappa_{z}\right)>1$ (Gurvich and Chunchuzov, 2008a, b) characterizes elongation of the 3-D spectrum along $\kappa_{z}$; it can be considered as the anisotropy coefficient, which depends on vertical wave number. The properties of 3-D and 1-D vertical and horizontal spectra obtained by integration of Eq. (2) over wave number plane are discussed in detail in Gurvich and Chunchuzov (2008a, b). The 1-D vertical spectrum does not depend on $\eta\left(\kappa_{z}\right)$ and has the -3 slope, well known from experimental data and theoretical models (e.g., Fritts and Alexander, 2003, and references therein). The 1-D horizontal spectrum corresponding to Eq. (2) is a function of $\eta\left(\kappa_{z}\right)$. It can contain domains with different slopes (Gurvich and Chunchuzov, 2008a, b). We will define $\eta\left(\kappa_{z}\right)$ later, based on experimental data.

The isotropic component $\Phi_{K}$ corresponds to the classical Kolmogorov's model of the locally isotropic turbulence (Monin and Yaglom, 1975):

$$
\begin{gathered}
\Phi_{K}(\kappa)=0.033 C_{K} \kappa^{-11 / 3} \exp \left(-\kappa^{2} / \kappa_{K}^{2}\right), \\
\kappa^{2}=\kappa_{x}^{2}+\kappa_{y}^{2}+\kappa_{z}^{2},
\end{gathered}
$$

with the slope $-5 / 3$ of the corresponding 1-D spectrum. For description of scintillations, $\Phi_{K}$ is characterized by two parameters: the structure characteristic $C_{K}$ and the inner scale $l_{K}=5.92 / \kappa_{K}$ (Tatarskii, 1971). Insufficient sampling frequency of the GOMOS photometers prevents reconstruction of $l_{K}$ from the scintillation spectra (Sofieva et al., 2007a, 2013). We will therefore use the diffraction approximation for modeling isotropic scintillation spectra, i.e., $l_{K} \ll \rho_{\mathrm{F}}=$ $\sqrt{2 \pi \lambda L} \approx 3.5 \mathrm{~m}$, where $\rho_{\mathrm{F}}$ is the Fresnel scale, $L$ is the distance from the ray perigee to the detector (for GOMOS, $L \approx 3225 \mathrm{~km}$ ).

\subsection{Approximations of phase screen and weak scintillations}

Due to exponential decrease of air density with altitude, the effective thickness of the atmosphere along the ray in occultation experiments is $L_{\mathrm{ef}}=\sqrt{\pi R_{\mathrm{E}} H_{0}} \approx 350 \mathrm{~km}$ (Gurvich and Brekhovskikh, 2001; Hubbard et al., 1978), where $R_{\mathrm{E}}$ is the Earth radius and $H_{0} \approx 6 \mathrm{~km}$ is the atmospheric scale height. This thickness is 10 times smaller than the distance $L$ between ray perigee and the satellite. The fact that the atmosphere is restricted to a thin layer (compared to the ray path from the atmosphere to a satellite), allows us to consider that only fluctuations of phase are formed at the exit from the atmosphere, while intensity fluctuations appear as a result of light interference during free-space propagation from the atmosphere to a detector (Gurvich et al., 2006; Hubbard et al., 1978). An equivalent phase screen, which models phase fluctuations at the exit from the atmosphere, is placed in the plane perpendicular to the incident rays and passing through 
the Earth center. Properties of the phase screen and its applicability to satellite experiments are discussed in detail in e.g., Gurvich and Brekhovskikh (2001), Gurvich (1984) and Hubbard et al. (1978). The limitation of phase fluctuations of light in the atmosphere due to changes in the orientation of anisotropic inhomogeneities along the line of sight when $\eta>\eta_{\mathrm{cr}}=\sqrt{R_{\mathrm{E}} / H_{0}} \approx 30$ (Gurvich and Brekhovskikh, 2001; Gurvich, 1984) is taken into account in our theoretical calculations.

The approximation of weak scintillations (normalized scintillation variance $\sigma_{I}^{2} \ll 1$ ) (Tatarskii, 1961, 1971) allows using simple linear relations between the 3-D wave number spectrum of atmospheric irregularities and the 2-D scintillation spectrum (Gurvich and Brekhovskikh, 2001; Gurvich and Kan, 2003b; Sofieva et al., 2007a). For low-orbit satellites, weak scintillations approximation is typically valid for altitudes above $30 \mathrm{~km}$ (Gurvich and Kan, 2003a; Sofieva et al., 2007a). Global distributions of scintillation variance observed from GOMOS are shown in Sofieva et al. (2007b). Note that due to the linearity, spectra of weak scintillations for the model (Eq. 1) can also be considered as the sum of statistically independent anisotropic and isotropic components of scintillations. The large velocity of line of sight in satellite experiments allows using the hypothesis of "frozenfield" irregularities for relation between temporal and spatial (wave number) scintillation spectra (for tangential occultations, the line-of-sight velocity around the minimal ray perigee altitude $h_{0}$ is practically equal to the Envisat velocity: $v_{\mathrm{h}} \approx v_{s}=7.45 \mathrm{~km} \mathrm{~s}^{-1}$ ).

The analysis of strong scintillations is a much more complicated problem. Complex nonlinear relationships between the parameters of atmospheric irregularities and scintillation are complicated further by the mutual influence of differenttype irregularities on strong scintillations (Gurvich et al., 2011). The properties of strong scintillation spectra in occultation experiments, as well as possibilities for reconstruction of atmospheric parameters are analyzed by numerical simulations and presented in Gurvich and Fedorova (2008) and Gurvich et al. (2006, 2011). The strong scintillations are not considered in our paper.

\section{Methodology for estimating atmospheric parameters}

\subsection{The scheme of observations in tangential occultations}

The scheme of tangential occultations is shown in Fig. 1. In the left panel, only the rays corresponding to the red photometer are shown for simplicity. The $x$ axis is along star to satellite direction, the $z$ axis is perpendicular to the rays. The phase screen (PS) is located in the plane $y O z$; the intersection points of the rays with the phase screen are marked. Tangential occultations with the minimal ray perigee altitude $h_{0}=0-100 \mathrm{~km}$ are observed by GOMOS if a star is located near the satellite orbital pole at an angular distance $\theta=28.4^{\circ}-25.5^{\circ}$ (Dalaudier et al., 2001; Gurvich and Kan, 2003a). The conditions for tangential occultations are realized seldom. The tangential occultations were performed with a dedicated mission planning in the year 2002. Altogether, 32 tangential occultations have been performed.

Figure 1, right, shows the trajectories of rays in the phase screen during tangential occultations. The blue and red light rays, which are recorded simultaneously by the detectors, are separated vertically in the atmosphere due to chromatic refraction. The chromatic shift $\Delta_{\mathrm{c}}$ can be determined using the known wavelength dependence of the refractive index and the air density, therefore $\Delta_{\mathrm{c}}$ can be determined with a sufficient accuracy for the current application using a standard atmosphere model (Dalaudier et al., 2001). Above $30 \mathrm{~km}$, change of $\Delta_{c}$ with height is similar to the change of the mean air density; below $30 \mathrm{~km}, \Delta_{\mathrm{c}}$ increases with a saturation as the altitude decreases. The chromatic shift $\Delta_{\mathrm{c}}$ is the main factor causing the de-correlation of scintillations at different wavelengths (Kan, 2004). For anisotropic irregularities, the ray trajectories around the minimal height $h_{0}$ can be considered as parallel to the Earth's horizon provided $\tan \alpha \geq \eta_{\max }(\alpha$ is the obliquity angle, i.e., the angle between the local vertical and the tangent to the ray trajectory in the phase screen). Assuming that the maximal anisotropy of irregularities $\eta_{\max } \leq 150$, then the parts of trajectories with $89.6^{\circ} \leq \alpha \leq 90^{\circ}$ extending $\sim 260 \mathrm{~km}$ each side from $h_{0}$ satisfy the condition of horizontality. The orbital precession leads to changes of the minimal height $h_{0}$ in successive orbits (orbit duration is $100 \mathrm{~min}$ ).

\subsection{Properties of the scintillation and description of the methodology}

Scintillations are strongly influenced by small-scale irregularities, whose contribution to the total scintillation variance is dominating. For the anisotropic component, these are irregularities with the vertical scales $\sim l_{\mathrm{W}}$. The values of $l_{\mathrm{W}}$, which are in the range of some tens of meters (Gurvich and Kan, 2003b; Sofieva et al., 2007a), exceed significantly the Fresnel scale $\rho_{\mathrm{F}}$, therefore diffractive effects are not important for anisotropic scintillations. Conversely, the diffractive effects are dominant for scintillations caused by isotropic irregularities if $\rho_{\mathrm{F}}>l_{K}$ (Tatarskii, 1971).

For tangential occultations, the characteristic scale of horizontal auto-spectra of anisotropic scintillations is given by the product $l_{\mathrm{h}, \mathrm{a}}=l_{\mathrm{W}} \eta\left(\kappa_{\mathrm{W}}\right)$. From auto-spectra only, it is impossible to retrieve both parameters $l_{\mathrm{W}}$ and $\eta\left(\kappa_{\mathrm{W}}\right)$. Using cross-spectra and coherency spectra of chromatic scintillation allows decomposing the horizontal coherency scale $l_{\mathrm{h}, \mathrm{c}}=2 \pi \Delta_{\mathrm{c}} \eta\left(\Delta_{\mathrm{c}}^{-1}\right)$ into its components: the vertical scale $2 \pi \Delta_{\mathrm{c}}$ and the anisotropy coefficient $\eta\left(\Delta_{\mathrm{c}}^{-1}\right)$. Indeed, the chromatic separation of rays $\Delta_{\mathrm{c}}$ is a vertical transition scale for the coherency: only irregularities with vertical scales larger than $\Delta_{\mathrm{c}}\left(\kappa_{z}^{-1} \geq \Delta_{\mathrm{c}}\right)$ can be crossed by both red and 

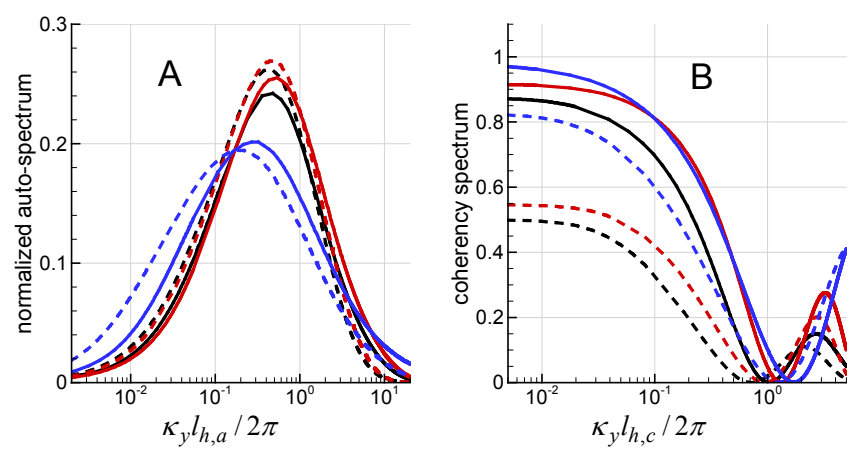

Figure 2. Theoretical auto-spectra (A) and coherency spectra (B) for altitudes $h_{0}=40 \mathrm{~km}$ (solid lines) and $h_{0}=34 \mathrm{~km}$ (dashed lines), for different models of the following atmospheric irregularities: black - the model of Gurvich and Brekhovskikh (2001) with the constant anisotropy, red - the model of Gurvich and Chunchuzov $(2008 \mathrm{a}, \mathrm{b})$ with $\eta\left(\kappa_{z}\right)=$ Const, blue - the model of Gurvich and Chunchuzov (2008a, b) with variable anisotropy. Autospectra are presented as the product of power spectral density multiplied by wave number and normalized by scintillation variance. On the $\mathrm{x}$-axes, the normalized wave numbers are $\kappa_{y} l_{\mathrm{h}, \mathrm{a}} / 2 \pi=$ $\kappa_{y} l_{\mathrm{W}} \eta\left(\kappa_{\mathrm{W}}\right) / 2 \pi$ for auto-spectra and $\kappa_{y} l_{\mathrm{h}, \mathrm{c}} / 2 \pi=\kappa_{y} \Delta_{\mathrm{c}} \eta\left(\Delta_{\mathrm{c}}^{-1}\right)$ for coherency spectra.

blue rays and therefore scintillations at these scales are coherent. For smaller scales, the chromatic scintillations are not coherent. For scintillations generated by anisotropic irregularities, the coherency level is defined by the ratio of the "correlating" scale $l_{\mathrm{W}}$ and the "de-correlating" scale $2 \pi \Delta_{\mathrm{c}}$ (Kan, 2004). For scintillations caused by isotropic irregularities, the "correlating" scale is $\rho_{\mathrm{F}}$ (or $l_{K}$ ) (Kan, 2004).

Due to different symmetry, contributions of anisotropic and isotropic irregularities in observed scintillations depend on obliquity angle $\alpha$ (the scanning direction of the atmosphere). With increasing $\alpha$, the temporal auto-spectrum of anisotropic scintillations is shifted toward lower frequencies, while the spectrum of isotropic scintillations is displaced to higher frequencies. Therefore, the estimates of isotropic component of scintillation can be obtained using the highfrequency part of scintillation spectra. The frequency separation of anisotropic and isotropic scintillations for oblique occultations has been applied in Gurvich and Kan (2003b) and Sofieva et al. (2007b). It is maximal for pure horizontal occultations $\left(\alpha=90^{\circ}\right)$, thus we will also use it for detection of isotropic component in measured scintillation spectra.

Figure 2 shows theoretical horizontal auto- and coherency scintillation spectra as a function of normalized wave numbers. Along with calculations for the anisotropicirregularities model with variable anisotropy (Gurvich and Chunchuzov, 2008a, b), we present also, for comparison, the spectra calculated using the model (Gurvich and Chunchuzov, 2008a, b) in which $\eta\left(\kappa_{z}\right)=$ Const is taken, as well as the spectra computed using the simpler model with constant anisotropy (Gurvich and Brekhovskikh, 2001). The auto-spectra are presented as a normalized product of power spectral density and horizontal wave number, in order to highlight the characteristic horizontal scale $l_{\mathrm{h}, \mathrm{a}}$ corresponding approximately to the maxima of spectra in this representation. The calculations are performed for the altitude $h_{0}=40 \mathrm{~km}$ with $\Delta_{\mathrm{c}}=2.2 \mathrm{~m}, l_{\mathrm{W}}=40 \mathrm{~m}$ and for the altitude $h_{0}=34 \mathrm{~km}$ with $\Delta_{\mathrm{c}}=5.4 \mathrm{~m}, l_{\mathrm{W}}=20 \mathrm{~m}$.

In case of variable anisotropy, $\eta\left(\kappa_{z}\right)$ increases from 1 at small scales of the order of a few decimeters up to 100 (with saturation) for the vertical scales $0.5-1.0 \mathrm{~km}$ (the used functional dependence $\eta\left(\kappa_{Z}\right)$ is presented below in Eq. 5). For calculations with the constant anisotropy model, $\eta_{0}$ is taken equal to $\eta\left(\kappa_{z}\right)$ for the scale corresponding to $\eta_{0}=$ $\eta\left(\kappa_{z}=\kappa_{\mathrm{W}}\right)$ for auto-spectra and $\eta_{0}=\eta\left(\kappa_{z}=\Delta_{\mathrm{c}}^{-1}\right)$ for coherency spectra. The characteristic horizontal scale of autospectra $l_{\mathrm{h}, \mathrm{a}}=l_{\mathrm{W}} \eta\left(\kappa_{\mathrm{W}}\right)$ is $1.6 \mathrm{~km}$ for $h_{0}=40 \mathrm{~km}\left(\eta\left(\kappa_{\mathrm{W}}\right)=\right.$ $40)$ and $0.5 \mathrm{~km}$ for $h_{0}=34 \mathrm{~km}\left(\eta\left(\kappa_{\mathrm{W}}\right)=25\right)$, while the horizontal scales of coherency spectra $l_{\mathrm{h}, \mathrm{c}}=2 \pi \Delta_{\mathrm{c}} \eta\left(\Delta_{\mathrm{c}}^{-1}\right)$ are $0.26 \mathrm{~km}$ and $1.2 \mathrm{~km}$, with corresponding anisotropy coefficients $\eta\left(\Delta_{\mathrm{c}}^{-1}\right)=20$ and $\eta\left(\Delta_{\mathrm{c}}^{-1}\right)=35$, respectively.

As observed in Fig. 2a, auto-spectra corresponding to the model with constant anisotropy are very close to each other. In case of variable anisotropy, increasing anisotropy at large scales (and reducing it at small scales) lead to a spread of the spectral peak in this representation and to some shift toward lower frequencies. This is due to the fact that the spectral density at moderate anisotropy increases with $\eta$ (for $100>\eta>30$, the spectral level increases with a saturation) (Gurvich and Brekhovskikh, 2001).

As noted above, the coherency level depends on the ratio of the inner scale $l_{\mathrm{W}}$ and the chromatic ray separation $\Delta_{\mathrm{c}}$. Therefore, coherency (for scales larger than $\Delta_{\mathrm{c}}$ ) is significantly higher at $40 \mathrm{~km}$ than that at $34 \mathrm{~km}$, for all models (Fig. 2b). In case of variable anisotropy, a significant increase of anisotropy towards large scales results in an increased coherency level. It should be noted that the normalization of wave numbers by the coherency scales brings together minima of coherency spectra, for all models. The difference in the positions of minima for different altitudes $34 \mathrm{~km}$ and $40 \mathrm{~km}$ does not exceed $30 \%$, for each model. A small systematic difference in coherency scale (i.e., the scale corresponding to the minimum of the coherency spectrum) for models with constant and variable anisotropy can be eliminated by introducing a corresponding coefficient. Noticeable oscillations in the coherency spectrum after the primary minimum are the result of idealization of the model spectrum of irregularities and of the adopted approximations.

Thus, the estimation of atmospheric parameters using horizontal chromatic scintillation spectra consists of the following procedures:

- Separation of isotropic and anisotropic components of a measured scintillation spectrum and estimating the parameters of Kolmogorov turbulence; 
- Estimation of the anisotropy coefficient $\eta\left(\Delta_{c}^{-1}\right)$ from the horizontal coherency scale, for each occultation;

- Selection of the functional dependence of the anisotropy coefficient $\eta\left(\kappa_{z}=\Delta_{\mathrm{c}}^{-1}\right)$ and estimation of its parameters;

- Estimating the parameters of the 3-D spectrum of anisotropic irregularities by fitting the modeled (theoretical) spectra to experimental spectra.

The procedure is started with the detection of the isotropic component, because its properties, as well as the properties of turbulence generating these scintillations, are well known (Monin and Yaglom, 1975; Tatarskii, 1971). The detection is based on the fact that the cutoff frequency of the spectrum of anisotropic scintillation, which is defined by the horizontal scale $l_{\mathrm{W}} \eta\left(\kappa_{\mathrm{W}}\right)$, is only a few Hertz, while the Nyquist frequency for GOMOS is $f_{\mathrm{N}}=500 \mathrm{~Hz}$. It can therefore be assumed that the high frequency region $f=450-500 \mathrm{~Hz}$ of experimental spectra is produced exclusively by the isotropic component. Then we can obtain the estimate of the structure characteristic of Kolmogorov turbulence $C_{K}$ in the diffractive approximation $\rho_{\mathrm{F}} \gg l_{K}$ using the high-frequency part of scintillation auto-spectrum (Gurvich and Kan, 2003b; Sofieva et al., 2007b). At altitudes $h_{0}<40 \mathrm{~km}, \rho_{\mathrm{F}}<2 \pi \Delta_{\mathrm{c}}$ and therefore coherency of isotropic scintillations is negligible. At higher altitudes $h_{0} \geq 40 \mathrm{~km}$, where $l_{K} \geq \rho_{\mathrm{F}}$ and is comparable with $2 \pi \Delta_{\mathrm{c}}$, one can estimate also the inner scale $l_{K}$ using the residual coherency of isotropic chromatic scintillations (Kan, 2004).

Using additivity of the components in weak scintillation spectra, we can obtain the estimates of anisotropic components for the horizontal auto-spectra $V_{\mathrm{a}}\left(\lambda_{\mathrm{B}} ; \kappa_{y}\right), V_{\mathrm{a}}\left(\lambda_{\mathrm{R}} ; \kappa_{y}\right)$, the cross-spectrum $V_{\mathrm{a}}\left(\lambda_{\mathrm{B}}, \lambda_{\mathrm{R}} ; \kappa_{y}\right)$ and the coherency spectrum $\operatorname{coh}_{a}\left(\lambda_{\mathrm{B}}, \lambda_{\mathrm{R}} ; \kappa_{y}\right)=\frac{\left|V_{\mathrm{a}}\left(\lambda_{\mathrm{B}}, \lambda_{\mathrm{R}} ; \kappa_{y}\right)\right|^{2}}{V_{\mathrm{a}}\left(\lambda_{\mathrm{B}} ; \kappa_{y}\right) V_{\mathrm{a}}\left(\lambda_{\mathrm{R}} ; \kappa_{y}\right)}$ by subtracting the fitted model for isotropic component from the measured (total) scintillation spectra. From the horizontal coherency scale $2 \pi \Delta_{\mathrm{c}} \eta\left(\Delta_{\mathrm{c}}^{-1}\right)$, which corresponds to the position of the primary minimum of the coherency spectrum, we can estimate the anisotropy coefficient $\eta$ corresponding to the vertical scale $2 \pi \Delta_{c}$, for each occultation.

In our analyses, we have used 12 occultations with altitudes $h_{0}$ from $30 \mathrm{~km}$ to $43 \mathrm{~km}$ and with corresponding scales $2 \pi \Delta_{\mathrm{c}}$ from $8 \mathrm{~m}$ to $55 \mathrm{~m}$ (see also details on the data in Sect. 4). The obtained estimates $\eta\left(\Delta_{\mathrm{c}}^{-1}\right)$ together with some assumptions about the anisotropy behavior outside the range of probed scales are then used for determination of the functional dependence $\eta\left(\kappa_{z}\right)$ and fitting of its parameters. Finally, the theoretical spectra of the anisotropic component with the selected dependence $\eta\left(\kappa_{z}\right)$ were fitted to the detected experimental auto- and coherency spectra of the anisotropic component. In this fitting, the values of the anisotropy coefficient $\eta\left(\Delta_{\mathrm{c}}^{-1}\right)$ are refined and the estimates of the structure characteristic $C_{\mathrm{W}}$ and characteristic of inner and outer scales for the spectra model of anisotropic irregularities are obtained.
In calculations of theoretical scintillation spectra for the GOMOS fast photometers, the effects related to the finite width of optical filters, finite integration time $\tau=1 \mathrm{~ms}$ and aliasing are taken into account. These effects are discussed in details in Gurvich and Brekhovskikh (2001), Kan (2004) and Kan et al. (2001). In this paper, for demonstrating the capabilities of the method, we have applied visual fitting.

\section{Experiment and comparison with model calculations}

Tangential occultations were not included in the routine program of GOMOS observations; they were carried out under a special scheme. Due to the limited azimuth tracking angle (Dalaudier et al., 2001), the measurements were performed on descending occultation leg and, in some cases, they covered also a small part of the ascending leg. From 32 tangential occultations found in the GOMOS database, twelve were selected for further analyses. We selected the occultations containing a sufficiently long (not less than $200 \mathrm{~km}$ ) horizontal path with $\tan \alpha \geq 150$ and satisfying the condition of weak scintillation $\left(\sigma_{I}^{2} \leq 0.5\right)$. All the tangential occultations have been performed in September-November 2002. Some information about these occultations is collected in Table 1. The minimal altitudes $h_{0}$ range from $43.4 \mathrm{~km}$ down to $30.5 \mathrm{~km}$, and the corresponding vertical scales $2 \pi \Delta_{\mathrm{c}}$ vary from $8 \mathrm{~m}$ to $55 \mathrm{~m}$, respectively.

The chromatic separation $2 \pi \Delta_{\mathrm{c}}$ is the main parameter in the considered problem; therefore occultations in Table 1 are sorted according to its increasing value (thus with decreasing altitude) for visualization. The duration of the horizontal sections ranges from $29 \mathrm{~s}$ to $50 \mathrm{~s}$ with lengths from $220 \mathrm{~km}$ to $370 \mathrm{~km}$ and a spatial sampling of $7.5 \mathrm{~m}$. Using these data samples, we computed Fast Fourier Transform (FFT) periodograms and averaged them using a spectral window with a variable width $\Delta f$ : the window has a constant quality $f / \Delta f=2$ at low frequencies and a constant width $\Delta f_{\max }=20-35 \mathrm{~Hz}$ at higher frequencies. Long scintillation records have allowed accumulating a large number of averaged periodograms $N$ : the spectral window of width $\Delta f_{\max }$ contained 1001 periodograms. Instrumental noise was removed from scintillation spectra by using the scintillation records at maximal ray perigee altitudes of $65-70 \mathrm{~km}$.

Figure $3 \mathrm{a}$ and $\mathrm{b}$ show experimental auto- and coherency spectra for $h_{0}=43.4 \mathrm{~km}$ (orbit 03438 ) and for $h_{0}=34.1 \mathrm{~km}$ (orbit 03818). The isotropic components in the scintillation auto-spectra were estimated using high-frequency parts of the experimental spectra. A significant difference in the spectral amplitudes of isotropic scintillations for blue and red photometers at $h_{0}=34.1 \mathrm{~km}$ is caused by different optical filter widths that reduces the amplitude of scintillations (Kan, 2004; Kan et al., 2001): the refractivity width, which is the vertical width of the light source projected onto the phase plane, is 2.5 times larger for the blue photometer. This broadening results from regular chromatic refraction combined 
Table 1. Information about the selected tangential occultations

\begin{tabular}{|c|c|c|c|c|c|c|c|c|c|}
\hline $\begin{array}{l}\text { Date and } \\
\text { orbit }\end{array}$ & star & $\begin{array}{r}\text { Min altit } \\
h_{0}, \mathrm{~km}\end{array}$ & $\begin{array}{l}\text { Coordinates } \\
\text { of } h_{0}\end{array}$ & $\begin{array}{l}\text { Chrom scale } \\
2 \pi \Delta_{\mathrm{c}}, \mathrm{m}\end{array}$ & $\begin{array}{l}\text { Variances } \\
\text { blue/red }\end{array}$ & $\begin{array}{r}C_{K} \times 10^{9} \\
\mathrm{~m}^{-2 / 3}\end{array}$ & $\begin{array}{r}C_{\mathrm{W}} \times 10^{11} \\
\mathrm{~m}^{-2}\end{array}$ & $\begin{array}{r}l_{\mathrm{W}} \\
\mathrm{m}\end{array}$ & $\begin{array}{r}\text { Anisotropy } \\
\eta\left(\Delta_{\mathrm{c}}^{-1}\right)\end{array}$ \\
\hline 27 Oct $2002 \# 03437$ & $\beta$ CMa Mirzam & 43.4 & $72^{\circ} \mathrm{N}, 14^{\circ} \mathrm{W}$ & 8.3 & $0.007 / 0.007$ & 3.5 & 1.0 & 40 & 13 \\
\hline 27 Oct $2002 \# 03438$ & $\beta$ CMa Mirzam & 43.4 & $72^{\circ} \mathrm{N}, 38^{\circ} \mathrm{W}$ & 8.3 & $0.008 / 0.007$ & 4.5 & 0.45 & 32 & 13 \\
\hline 27 Oct $2002 \# 03436$ & $\beta$ CMa Mirzam & 43.3 & $72^{\circ} \mathrm{N}, 10^{\circ} \mathrm{E}$ & 8.4 & $0.006 / 0.006$ & 2.5 & 0.71 & 40 & 11 \\
\hline 27 Oct $2002 \# 03439$ & $\beta$ CMa Mirzam & 43.3 & $72^{\circ} \mathrm{N}, 64^{\circ} \mathrm{W}$ & 8.4 & $0.008 / 0.007$ & 4.0 & 0.32 & 25 & 13 \\
\hline 28 Oct $2002 \# 03455$ & $\beta$ CMa Mirzam & 42.4 & $72^{\circ} \mathrm{N}, 100^{\circ} \mathrm{W}$ & 9.7 & $0.04 / 0.03$ & 17.5 & 0.52 & 10 & 11 \\
\hline 22 Nov 2002 \#03819 & $\alpha$ Hyd Alphard & 36.8 & $39^{\circ} \mathrm{N}, 41^{\circ} \mathrm{E}$ & 21.7 & $0.08 / 0.09$ & 2.3 & 0.78 & 16 & 37 \\
\hline 19 Sep $2002 \# 02893$ & $\delta$ Ori Mintaka & 36.2 & $17^{\circ} \mathrm{N}, 70^{\circ} \mathrm{W}$ & 24.0 & $0.05 / 0.07$ & 3.5 & 1.3 & 30 & 27 \\
\hline 19 Sep $2002 \# 02893$ & $\beta$ Ori Rigel & 34.6 & $35^{\circ} \mathrm{N}, 70^{\circ} \mathrm{W}$ & 30.2 & $0.20 / 0.31$ & 10.0 & 1.1 & 5 & 27 \\
\hline 22 Nov $2002 \# 03818$ & $\alpha$ Hyd Alphard & 34.1 & $39^{\circ} \mathrm{N}, 66^{\circ} \mathrm{E}$ & 32.5 & $0.13 / 0.14$ & 2.4 & 0.78 & 12 & 51 \\
\hline 19 Sep $2002 \# 02892$ & $\delta$ Ori Mintaka & 32.9 & $17^{\circ} \mathrm{N}, 45^{\circ} \mathrm{W}$ & 38.7 & $0.07 / 0.09$ & 1.0 & 0.78 & 30 & 45 \\
\hline 22 Nov $2002 \# 03817$ & $\alpha$ Hyd Alphard & 31.6 & $38^{\circ} \mathrm{N}, 91^{\circ} \mathrm{E}$ & 47.6 & $0.36 / 0.56$ & 5.4 & 0.91 & 5 & 50 \\
\hline 24 Oct $2002 \# 03395$ & $\alpha$ CMa Sirius & 30.5 & $63^{\circ} \mathrm{N}, 80^{\circ} \mathrm{W}$ & 55.3 & $0.29 / 0.48$ & 1.5 & 6.5 & 20 & 51 \\
\hline
\end{tabular}
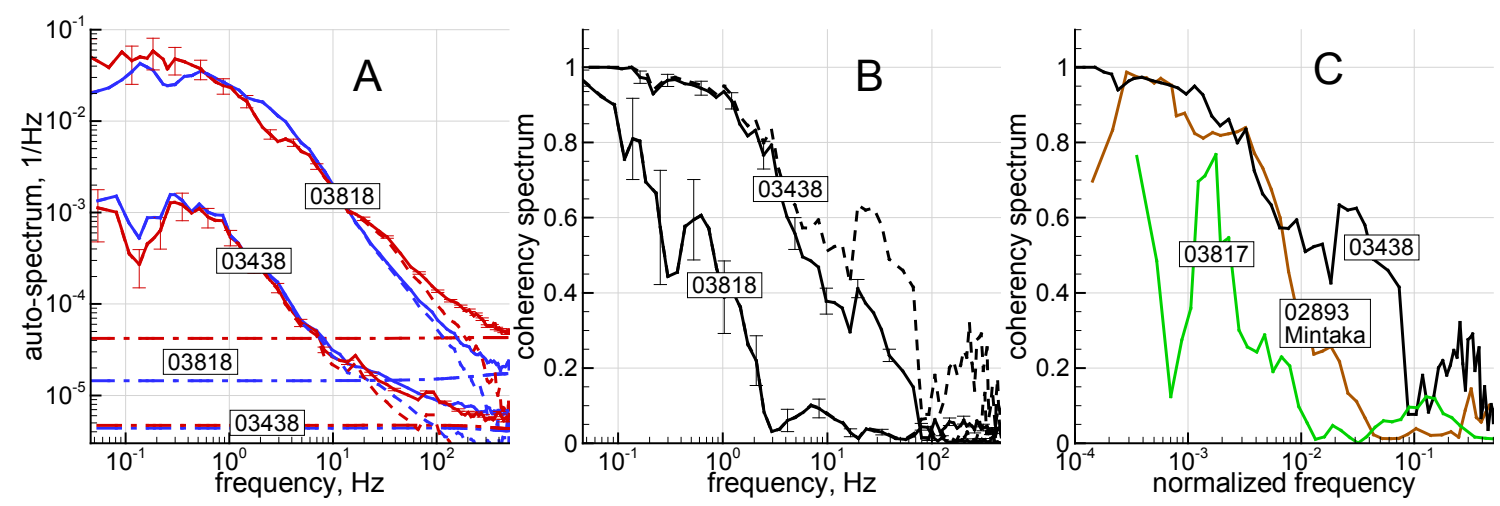

Figure 3. (A) experimental auto-spectra and detected isotropic and anisotropic components of these spectra. Solid lines: experimental spectra, dot-dashed lines: estimates of the isotropic component, dashed lines: estimates of the anisotropic component. Colors of the lines in (A) correspond to wavelengths of the photometers. Error bars show rms uncertainty of the spectral density estimates (error bars are shown only for the red-photometer auto-spectra; for the blue photometer, uncertainties are the same). (B) coherency spectra of measured scintillations (solid lines) and of anisotropic component (dashed lines). (C) coherency spectra of the anisotropic scintillation component presented as a function of the normalized frequency $\frac{2 \pi f}{v_{\mathrm{h}}} \Delta_{\mathrm{c}}$, for different values of the chromatic shift. The numbers in boxes are the orbit numbers.

with the finite bandwidth of the optical filter (Dalaudier et al., 2001). At the altitude $h_{0}=43.4 \mathrm{~km}$, where the refractive widths of optical filters are smaller than the Fresnel scale, the influence of chromatic smoothing is very small. Relative rms errors in auto-spectra estimates are inversely proportional to $\sqrt{N}$, while for coherence spectra, they are also inversely proportional to $\sqrt{\mathrm{coh}}$ (Bendat and Piersol, 1986). Accordingly, the uncertainty of the estimated coherency spectra is significantly larger than for the auto-spectra, especially for low coherency values.

As discussed above, the anisotropic component of scintillations dominates at low frequencies. If the characteristic coherency scale belongs to this frequency range, then the coherency spectrum of the anisotropic component practically coincides with the full coherency spectrum of the measured scintillations (as for the orbit 3818 in Fig. 3b). This situation is quite typical for all occultations with altitudes $h_{0} \leq 36 \mathrm{~km}$ due to increased chromatic shift and a more efficient damping the isotropic component by optical filters at lower altitudes (Kan, 2004). We emphasize that the characteristic frequency of the coherence spectrum corresponding to the position of its minimum for $h_{0}=43.4 \mathrm{~km}$ (orbit 03438) in Fig. 3b is about 30 times larger than the corresponding frequency for $h_{0}=34.1 \mathrm{~km}$ (orbit 03818), while the chromatic separation scale $2 \pi \Delta_{\mathrm{c}}$ is only 4 times smaller.

The estimates of the structure characteristic of Kolmogorov turbulence $C_{K}$, which are retrieved from autospectra of the isotropic component of scintillation, are presented in Table 1. They are in agreement with the previous estimates by Gurvich and Kan (2003b) and Sofieva et al. (2007a). Also, for occultations with $h_{0} \approx 43 \mathrm{~km}$ the estimates of the inner turbulence scale $l_{K}$ are obtained using the residual coherency of isotropic scintillations (Kan, 2004). The obtained values are $l_{K} \approx 2.0-4.0 \mathrm{~m}$, in agreement with the estimates of Gurvich and Kan (2003b), which were 
obtained directly from auto-spectra of scintillations recorded with a higher sampling rate of $8-16 \mathrm{kHz}$.

Figure $3 \mathrm{c}$ shows examples of coherency spectra for the three orbits: $03438,02893,03817$ with $2 \pi \Delta_{c}$ equal to $8.3 \mathrm{~m}, 24.0 \mathrm{~m}$ and $47.6 \mathrm{~m}$, respectively. The abscissa is the frequency normalized by $f^{*}=v_{\mathrm{h}} / 2 \pi \Delta_{\mathrm{c}}$, i.e., $f / f^{*}=$ $\frac{2 \pi f}{v_{\mathrm{h}}} \Delta_{\mathrm{c}}=\kappa_{y} \Delta_{\mathrm{c}}$. In this representation, the position of the minimum of the coherency spectrum is inversely proportional to the anisotropy coefficient $\eta\left(\Delta_{\mathrm{c}}^{-1}\right)$ (see Fig. 2b for comparison). As observed in Fig. $3 \mathrm{c}$, as the scale $2 \pi \Delta_{\mathrm{c}}$ increases, the corresponding anisotropy also increases: from $\sim 10$ for the orbit 03438 up to 50 for the orbit 03817 .

Preliminary estimates of the characteristic horizontal scales of coherency spectra for occultations presented in Table 1 showed that the anisotropy coefficient increases approximately linearly with the vertical scale in the range 8.3$55.3 \mathrm{~m}$. As a working hypothesis on asymptotic behavior of the anisotropy coefficient outside this range of scales, we assume that anisotropy continues to decrease at small scales and $\eta \rightarrow 1$ for scales of the order of a few decimeters; for large scales (a few hundred meters or more), $\eta$ gradually saturates to $\eta_{\max } \approx 100$. Then the dependence of anisotropy on the vertical wave number can be written in the simplest form as follows:

$\eta\left(\kappa_{z}\right)=\eta_{\min }+\frac{\eta_{\max }-\eta_{\min }}{1+\left|\kappa_{z}\right| / \kappa_{*}}$

where, according to our assumptions, $\eta_{\min }=1, \eta_{\max }=100$, while $\kappa_{*}>0$ is the (single) parameter to be fitted using the experimental estimates of the anisotropy coefficient.

The initial estimates $\tilde{\eta}\left(\Delta_{\mathrm{c}}^{-1}\right)$ obtained from characteristic scales of coherency spectra were used to determine the functional dependence $\tilde{\eta}\left(\kappa_{z}\right)$ based on Eq. (4). This dependence was then used for fitting the theoretical scintillation spectra to experimental spectra. At this stage, the estimates of the anisotropy coefficient $\eta\left(\Delta_{\mathrm{c}}^{-1}\right)$ are refined, and the estimates of the structure characteristic of anisotropic component as well as the inner and the outer scales are obtained. The refined estimates $\eta\left(\Delta_{\mathrm{c}}^{-1}\right)$ are used for determining the parameter $\kappa_{*}$ in the function $\eta\left(\kappa_{z}\right)$, Eq. (4).

In this procedure, for each occultation we get an estimate of the anisotropy coefficient $\eta$, for a specific wave number $\kappa_{z}=\Delta_{\mathrm{c}}^{-1}$. Because of the limited number of measurements, in order to determine the functional dependence of anisotropy $\eta\left(\kappa_{z}\right)$, we had to combine the occultation data held in different conditions (altitude, latitude, orography) and assume that they are random samples from the same statistical ensemble.

Figure $4 \mathrm{a}$ shows the dependence of the anisotropy coefficient $\eta$ on wave number $\kappa_{z}$ and the corresponding vertical scale $2 \pi / \kappa_{z}$ (the upper abscissa). The triangles indicate experimental values $\eta\left(\Delta_{\mathrm{c}}^{-1}\right)$, which are colored according to latitudes. Brown rectangles show other known estimates of anisotropy of small-scale (from a few meters to a few tens of meters) stratospheric irregularities (see figure caption).
These previous estimates support the hypothesis of weak altitude dependence of the anisotropy coefficient. Indeed, the estimates of Gurvich and Kon (1993) obtained using the angular dependence of backscatter radar signal give $\eta=10$ for $3.2 \mathrm{~m}$ scale at $20 \mathrm{~km}$ (the chromatic scale $2 \pi \Delta_{\mathrm{c}}=3.2 \mathrm{~m}$ corresponds to an altitude of $\sim 50 \mathrm{~km}$ ); the estimate of Kan et al. (2012) obtained from scintillations of a double star give $\eta=15-20$ for scales $10-20 \mathrm{~m}$ at $38-30 \mathrm{~km}$ (the chromatic scale of $10-20 \mathrm{~m}$ corresponds to altitudes $42-37 \mathrm{~km}$ ). The black line shows the function (Eq. 4), which approximates our experimental data with additional assumptions on asymptotics at small and large scales. The value of the parameter in Eq. (4) estimated using the least squares method is $\kappa_{*}=0.12 \mathrm{rad} \mathrm{m}^{-1}$. The limited data set does not allow making any definite assumptions about the latitudinal peculiarities of anisotropy. We can note, however, that the main assumption about increasing anisotropy with increasing scale of irregularities is confirmed by measurements for each latitude zone.

Figure $4 \mathrm{~b}$ and $\mathrm{c}$ compare experimental and theoretical auto- and coherency spectra of anisotropic scintillations for three occultations. As observed, theoretical spectra reasonably describe experimental ones. One can note that the high-frequency parts of theoretical auto-spectra decay more rapidly at high frequency than that of experimental ones. This is well seen on the auto-spectra for the orbit 03817, which are presented in a larger range of frequency and spectral density. Strong growth of anisotropy leads to an increase of auto- and coherency spectra at large scales, and, in particular, to the manifestation of the outer scale $L_{\mathrm{W}}$ in the low-frequency part of the horizontal scintillation spectra, in contrast to the model with constant anisotropy (Gurvich and Brekhovskikh, 2001; Gurvich and Kan, 2003a). One might suggest that this outer scale is related to the transition from saturated to unsaturated gravity waves and, consequently, it can also be marked by some transition for the anisotropy coefficient.

The obtained estimates of the structure characteristic $C_{\mathrm{W}}$, inner scale $l_{\mathrm{W}}$ and the anisotropy coefficient $\eta\left(\Delta_{\mathrm{c}}^{-1}\right)$ are collected in Table 1 . The estimates of the outer scale $L_{\mathrm{W}}$ are in the range $0.5-1.5 \mathrm{~km}$. The values of the structure characteristic and the inner scale are obtained using the spectral density amplitude and the high-frequency decay; they agree well with those reconstructed from vertical and oblique occultations (Gurvich and Kan, 2003b; Sofieva et al., 2009), despite the difference in the 3-D model of $\Phi_{\mathrm{W}}$ used in fitting the parameters. This can be partially explained by the above-mentioned saturation of scintillation amplitude with increasing anisotropy when $\eta>\approx 30$ (in reconstruction of GW spectra parameters in Gurvich and Kan, 2003b; Sofieva et al., 2009, the constant anisotropy $\eta=30$ was used). 

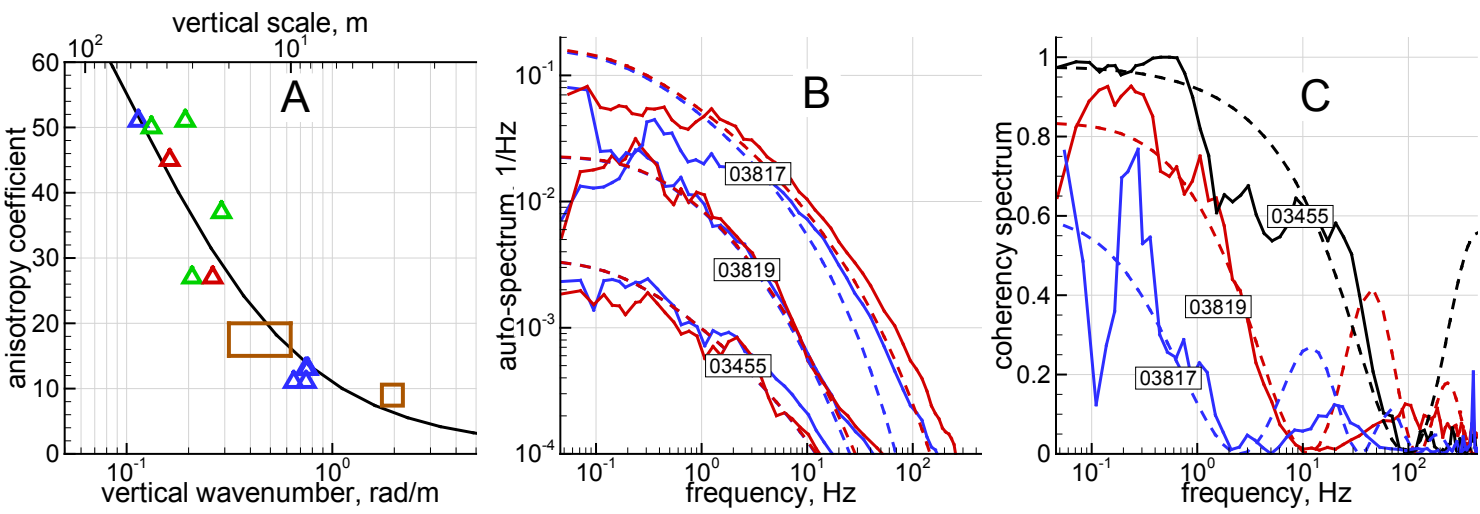

Figure 4. (A) the anisotropy coefficient. Experimental estimates are shown by triangles: blue for high latitude, green for mid-latitudes, red for tropics. Black line: approximation Eq. (5) of the experimental estimates. Brown square: the estimates of Gurvich and Kon (1993) using the angular dependence of backscatter radar signal for an altitude $\sim 20 \mathrm{~km}$; brown rectangle: the estimate of Kan et al. (2012) obtained from the analyses of chromatic scintillations of a double star for altitudes $30-38 \mathrm{~km}$. (B): autospectra and (C): coherency spectra of the anisotropic component of the anisotropic component. Solid lines: experimental spectra, dashed line: the fitted model spectra. The numbers in boxes are the orbit numbers.

\section{Discussion and summary}

In this paper, we have discussed the possibility of using stellar scintillations in specific tangential occultations for studying the structure of air density irregularities, mainly, for investigation of anisotropy of stratospheric irregularities. In tangential occultations, around the minimal altitude, light rays scan the atmosphere practically parallel to the horizon over several hundred kilometers. Due to chromatic refraction, the rays of different color are separated vertically by the chromatic shift $\Delta_{c}$, which can be evaluated using the model of standard atmosphere. We have used the data that satisfy the condition of weak scintillations. The data analyses have been performed using the two-component model of the 3-D spatial spectrum of atmospheric irregularities and the phase screen approximation. The method for anisotropy estimation is based on the fact that the horizontal coherency scale, which can be determined from measurements, is proportional to the product of the chromatic shift and the anisotropy coefficient, for weak scintillations.

One limitation of using chromatic scintillations is a larger uncertainty of cross- and coherency spectra estimates compared to auto-spectra of scintillations (Bendat and Piersol, 1986). In the considered measurements, this limitation is partially compensated by large statistical averaging of periodograms, due to long realizations (several hundred kilometers) with a high resolution (less than $10 \mathrm{~m}$ ) of measurements.

From each tangent occultation, we can get an anisotropy estimate only for a single altitude $h_{0}$ for the corresponding value of the wave number $\kappa_{z}=\Delta_{\mathrm{c}}^{-1}$. These estimates obtained for irregularities with vertical scales $8-55 \mathrm{~m}$ show that anisotropy grows with scale approximately from 10 to 50 . The limited data set (12 occultations) does not allow investigation of anisotropy structure in different observation conditions (altitude, latitude, season etc.). Therefore, for finding the functional dependence $\eta\left(\kappa_{z}\right)$ we had to assume its "universality" by considering that all measurements are samples from the same statistical ensemble. In other words, we assume that the anisotropy coefficient is a function of wave number but is not a function of altitude or latitude (or anything else). In addition, since the experimental anisotropy estimates are obtained in a limited range of scales, we assume the shape of the functional dependence (Eq. 4) and asymptotic behavior of anisotropy at large and small scales. The significant number of limitations and assumptions that were made in determining functional dependence of anisotropy implies that Eq. (4) should be regarded as a preliminary approximation of $\eta\left(\kappa_{z}\right)$.

We would like to note, however, that the available experimental data (from this and also from previous studies) agree well with the found approximation of $\eta\left(\kappa_{z}\right)$. They also support the assumption of weak dependence of the anisotropy coefficient on altitude, as discussed above. One more fact supports this assumption. For each occultation, in order to determine the inner scale $l_{\mathrm{W}}$ (which can differ significantly from the chromatic scale $2 \pi \Delta_{c}$ at the same altitude), we use the anisotropy estimates obtained in other tangent occultations (at different altitudes). The good agreement of the estimates $l_{\mathrm{W}}$ obtained in tangent occultations with that obtained from numerous vertical and oblique occultations (Alexandrov et al., 1990; Gurvich and Kan, 2003a, b; Sofieva et al., 2007a, 2009) also supports the validity of the assumption that anisotropy depends weakly on altitude in the range $20-45 \mathrm{~km}$.

It is important to notice that, according our analysis, anisotropy changes rapidly in the range of vertical scales 10$50 \mathrm{~m}$, which correspond to the scales of GW breaking. This is quite expected that the GW breaking induces changes in 
anisotropy. Another interesting observation is that variable anisotropy reaches rather large values $\sim 50$ at vertical scales of $\sim 50 \mathrm{~m}$, thus allowing the hypothesis that anisotropy saturates at scales of a few hundred meters and larger.

Estimates of dominant horizontal and vertical wavelengths of gravity waves have been obtained using satellite and radiosonde data (Alexander et al., 2008; Ern and Preusse, 2012; Ern et al., 2004; Wang and Alexander, 2010; Wang et al., 2005). Despite different observation methods and different data analyses, all these data indicate a very large anisotropy of large-scale structures: from a hundred to several hundreds. These results are in agreement with the theoretical estimates of anisotropy for large-scale GWs: $\eta \approx\left(N_{\mathrm{BV}} / f_{\mathrm{C}}\right) M^{-1}\left(N_{\mathrm{BV}}\right.$ is the Brunt-Väisälä frequency and $f_{\mathrm{C}}$ is the Coriolis parameter), with $M=1, \eta=100-200$ (Fritts and Alexander, 2003) and also with $M=0.3-0.4, \eta=250-500$ (Chunchuzov, 2002).

The previously obtained estimates of anisotropy for smallscale GWs (Gurvich and Kon, 1993; Kan et al., 2012) have shown that a significant anisotropy is also present at small scales, but it is much smaller than that for the large scales. The estimates of anisotropy, which are obtained in the present work for irregularities with vertical scales of 8-55 m are in agreement with previous data on small-scale structure and confirm the general assumption of variable anisotropy increasing with scale.

To compare the theory with the experiment and to obtain quantitative estimates of the parameters of the anisotropic component of atmospheric inhomogeneities, we have used a 3-D model of the GW spectrum with variable anisotropy, which depends on vertical wave number (Gurvich and Chunchuzov, 2008a, b). Considering the widespread use of the model with constant anisotropy in the scintillation analyses (Gurvich and Brekhovskikh, 2001; Gurvich and Kan, 2003b; Sofieva et al., 2007a), we have presented also a comparison of horizontal scintillation spectra for the models with constant and variable anisotropy. A detailed comparative analysis of these models is the subject of a separate study (some comparisons for different models are presented in Fedorova, 2012). Here we note only that the levels of coherency spectra, which depend on the ratio between the inner scale and the chromatic shift, can noticeably differ for various models, as seen in Fig. 2b. Therefore, we have not applied the method for estimating the inner scale (and then the anisotropy coefficient) using the coherency spectrum level proposed in Kan (2004).

Finally, let us discuss briefly a possibility for extension of the range of the investigated anisotropy scales. The scale of chromatic separation of rays, for which we estimate anisotropy, is defined by wavelengths and altitude $h_{0}$. The maximal height (or the minimal vertical scale) depends on brightness of light source, relative weights of anisotropic and isotropic components and on technical parameters of photometers (instrumental noise, sampling rate, filter width). One can expect that for photometers analogous to GOMOS, the upper altitude will be $\sim 50 \mathrm{~km}$ with the corresponding vertical chromatic separation scale $2 \pi \Delta_{\mathrm{c}} \approx 3.5 \mathrm{~m}$. The lower altitude limit is defined by the condition of weak scintillations (for low-orbit satellite, $h_{0} \approx 30 \mathrm{~km}$ and $2 \pi \Delta_{\mathrm{c}} \approx 60 \mathrm{~m}$ ). The linear relations between the spectra of weak scintillations and the spectra of atmospheric irregularities significantly simplify the data analyses and reconstruction of the parameters of atmospheric irregularities.

An increased interest to the occultation scintillation methods and the need for interpretation of data at lower altitudes have stimulated recent new research on strong scintillations (Gurvich and Fedorova, 2008; Gurvich et al., 2006, 2011). The results obtained in these studies do give hope for significant progress in this direction. Let us notice that the chromatic shift $2 \pi \Delta_{c}$ for GOMOS photometers at $h_{0}=20 \mathrm{~km}$ is about $200 \mathrm{~m}$. Adding a third-wavelength photometer and shifting the blue photometer to shorter wavelengths (refractivity is inversely proportional to squared wavelength of light) could also expand the opportunities for studies using chromatic scintillations. With the use of 3 carefully selected wavelengths, each tangent altitude would provide 3 distinct chromatic separations.

In conclusion, we note that the spectra of chromatic scintillations in tangential occultations allow studying anisotropy of small-scale stratospheric irregularities. The obtained anisotropy estimates for the scales of $8-55 \mathrm{~m}$ (the scales close to the buoyancy scale) indicate that variable anisotropy increases with the scale of atmospheric inhomogeneities.

Acknowledgements. The authors thank A. S. Gurvich, V. V. Vorobiev, and I. P. Chunchuzov for useful discussions and comments. The authors thank ESA, ACRI-ST and the GOMOS team for the GOMOS data. The work of V. Kan was supported by Russian Foundation for Basic Research, grant 12-05-00335. The work of V. F. Sofieva was supported by the Academy of Finland (projects MIDAT and ASTREX).

Edited by: C. von Savigny

\section{References}

Alexander, M. J., Gille, J., Cavanaugh, C., Coffey, M., Craig, C., Eden, T., Francis, G., Halvorson, C., Hannigan, J., Khosravi, R., Kinnison, D., Lee, H., Massie, S., Nardi, B., Barnett, J., Hepplewhite, C., Lambert, A., and Dean, V.: Global estimates of gravity wave momentum flux from high resolution dynamics limb sounder (HIRDLS) observations, J. Geophys. Res., 113, D15S18, doi:10.1029/2007JD008807, 2008.

Alexandrov, A. P., Grechko, G. M., Gurvich, A. S., Kan, V., Manarov, M. K., Pakhomov, A. I., Romanenko, Y. V., Savchenko, S. A., Serova, S. I., and Titov, V. G.: Spectra of temperature variations in the stratosphere as indicated by satellite-borne observation of the twinkling of stars, Izv. Atmos. Ocean. Phys., 26, 1-8, 1990.

Bendat, J. S. and Piersol, A. G.: Random Data: Analysis and Measurement Procedures, 2nd Edn., J. Wiley, New York, 1986. 
Chunchuzov, I. P.: On the high-wavenumber form of the Eulerian internal wave spectrum in the atmosphere, J. Atmos. Sci., 59, 1753-1772, 2002.

Dalaudier, F. and Gurvich, A. S.: A scalar three-dimensional spectral model with variable anisotropy, J. Geophys. Res., 102, 19449-19459, doi:10.1029/97JD00962, 1997.

Dalaudier, F., Kan, V., and Gurvich, A. S.: Chromatic refraction with global ozone monitoring by occultation of stars. I. Description and scintillation correction, Appl. Opt., 40, 866-877, 2001.

Ern, M. and Preusse, P.: Gravity wave momentum flux spectra observed from satellite in the summertime subtropics: Implications for global modeling, Geophys. Res. Lett., 39, L15810, doi:10.1029/2012GL052659, 2012.

Ern, M., Preusse, P., Alexander, M. J., and Warner, C. D.: Absolute values of gravity wave momentum flux derived from satellite data, J. Geophys. Res., 109, D20103, doi:10.1029/2004JD004752, 2004.

Ern, M., Preusse, P., Gille, J. C., Hepplewhite, C. L., Mlynczak, M. G., Russell III, J. M., and Riese, M.: Implications for atmospheric dynamics derived from global observations of gravity wave momentum flux in stratosphere and mesosphere, J. Geophys. Res., 116, D19107, doi:10.1029/2011JD015821, 2011.

Fedorova, O. V: Scintillation spectra behind inhomogeneties with variable anisotropy, Atmos. Ocean Opt., 25, 317-320, 2012.

Fritts, D. C. and Alexander, M. J.: Gravity wave dynamics and effects in the middle atmosphere, Rev. Geophys., 41, 1003, doi:10.1029/2001RG000106, 2003.

Gurvich, A. S.: Fluctuations in the observations of extraterrestrial cosmic sources through the earth's atmosphere, Radiophys. Quantum Electron., 27(8), 665-672, doi:10.1007/BF01040469, 1984.

Gurvich, A. S.: A heuristic model of three-dimensional spectra of temperature inhomogeneities in the stably stratified atmosphere, Ann. Geophys., 15, 856-869, doi:10.1007/s00585-997-0856-x, 1997.

Gurvich, A. S. and Brekhovskikh, V.: A study of turbulence and inner waves in the stratosphere based on the observations of stellar scintillations from space: A model of scintillation spectra, Waves in Random Media, 11, 163-181, 2001.

Gurvich, A. S. and Chunchuzov, I. P.: Parameters of the fine density structure in the stratosphere obtained from spacecraft observations of stellar scintillation, J. Geophys. Res., 108, 4166, doi:10.1029/2002JD002281, 2003.

Gurvich, A. S. and Chunchuzov, I. P.: Model of the ThreeDimensional Spectrum of Anisotropic Temperature Irregularities in a Stably Stratified Atmosphere, Izv. Atmos. Ocean. Phys., 44, 567-582, doi:10.1134/S0001433808050034, 2008a.

Gurvich, A. S. and Chunchuzov, I. P.: Three-dimensional spectrum of temperature fluctuations in stably stratified atmosphere, Ann. Geophys., 26, 2037-2042, doi:10.5194/angeo-26-20372008, 2008b.

Gurvich, A. S. and Fedorova, O. V: Reconstruction of turbulent parameters under strong scintillations, Atmos. Ocean Opt., 21, 96101,2008

Gurvich, A. S. and Kan, V.: Structure of air density irregularities in the stratosphere from spacecraft observations of stellar scintillation: 1. Three-dimensional spectrum model and recovery of its parameters, Izv. Atmos. Ocean. Phys., 39, 300-310, 2003 a.
Gurvich, A. S. and Kan, V.: Structure of air density irregularities in the stratosphere from spacecraft observations of stellar scintillation: 2. Characteristic scales, structure characteristics, and kinetic energy dissipation, Izv. Atmos. Ocean. Phys., 39, 311-321, 2003b.

Gurvich, A. S. and Kon, A. I.: Aspect sensitivity of radar returns from anisotropic turbulent irregularities, J. Electromagn. Waves Appl., 7, 1343-1353, 1993.

Gurvich, A. S., Dalaudier, F., and Sofieva, V. F.: Study of stratospheric air density irregularities based on two-wavelength observation of stellar scintillation by Global Ozone Monitoring by Occultation of Stars (GOMOS) on Envisat, J. Geophys. Res., 110, D11110, doi:10.1029/2004JD005536, 2005.

Gurvich, A. S., Vorob'ev, V. V and Fedorova, O. V: Determination of parameters of the spectrum of internal waves in the stratosphere from space-based observations of strong stellar scintillation, Izv. Atmos. Ocean. Phys., 42, 463-473, doi:10.1134/S0001433806040062, 2006.

Gurvich, A. S., Sofieva, V. F., and Dalaudier, F.: Global distribution of CT2 at altitudes $30-50 \mathrm{~km}$ from space-borne observations of stellar scintillation, Geophys. Res. Lett., 34, L24813, doi:10.1029/2007GL031134, 2007.

Gurvich, A. S., Vorob'ev, V. V., and Fedorova, O. V: Strong Scintillation Spectra behind the Atmosphere with Large- and SmallScale Inhomogeneities, Atmos. Ocean. Opt., 24, 347-357, 2011.

Hubbard, W. B., Jokipii, J. R., and Wilking, B. A.: Stellar occultation by turbulent planetary atmospheres: a wave-optical theory including a finite scale height, Icarus, 34, 374-395, 1978.

Hubbard, W. B., Lellouch, E., and Sicardy, B.: Structure of Scintillations in Neptune's Occultation Shadow, Astron. J., 325, 490502, 1988.

Kan, V.: Coherence and correlation of chromatic stellar scintillations in a space-borne occultation experiment, Izv. Atmos. Ocean. Opt., 17, 725-735, 2004.

Kan, V., Dalaudier, F., and Gurvich, A. S.: Chromatic refraction with global ozone monitoring by occultation of stars. II. Statistical properties of scintillations, Appl. Opt., 40, 878-889, 2001.

Kan, V., Sofieva, V. F., and Dalaudier, F.: Anisotropy of small-scale stratospheric irregularities retrieved from scintillations of a double star $\alpha$-Cru observed by GOMOS/ENVISAT, Atmos. Meas. Tech., 5, 2713-2722, doi:10.5194/amt-5-2713-2012, 2012.

Monin, A. S. and Yaglom, A. M.: Statistical Fluid Mechanics, Vol. 2, MIT Press: Cambridge, Massachusetts, 1975.

Raynaud, E., Matcheva, K., Drossart, P., Roques, F., and Sicardy, B.: A re-analysis of the 1971 Beta Scorpii occultation by Jupiter: study of temperature fluctuations and detection of wave activity, Icarus, 168, 324-335, doi:10.1016/j.icarus.2003.10.021, 2004.

Sofieva, V. F., Gurvich, A. S., and Dalaudier, F.: Gravity wave spectra parameters in 2003 retrieved from stellar scintillation measurements by GOMOS, Geophys. Res. Lett., 36, L05811, doi:10.1029/2008GL036726, 2009.

Sofieva, V. F., Gurvich, A. S., Dalaudier, F., and Kan, V.: Reconstruction of internal gravity wave and turbulence parameters in the stratosphere using GOMOS scintillation measurements, J. Geophys. Res., 112, D12113, doi:10.1029/2006JD007483, 2007a.

Sofieva, V. F., Kyrölä, E., Hassinen, S., Backman, L., Tamminen, J., Seppälä, A., Thölix, L., Gurvich, A. S., Kan, V., Dalaudier, F., Hauchecorne, A., Bertaux, J.-L., Fussen, D., Vanhellemont, 
F., Fanton D’Andon, O., Barrot, G., Mangin, A., Guirlet, M., Fehr, T., Snoeij, P., Saavedra, L., Koopman, R., and Fraisse, R.: Global analysis of scintillation variance: Indication of gravity wave breaking in the polar winter upper stratosphere, Geophys. Res. Lett., 34, L03812, doi:10.1029/2006GL028132, 2007 b.

Sofieva, V. F., Dalaudier, F., and Vernin, J.: Using stellar scintillation for studies of turbulence in the Earth's atmosphere, Philos. Trans. R. Soc. A Math. Phys. Eng. Sci., 371, 20120174 , doi:10.1098/rsta.2012.0174, 2013.

Tatarskii, V. I.: Wave propagation in a turbulent medium, edited by: Silverman, R. A., McGraw-Hill, New York, 1961.
Tatarskii, V. I.: The Effects of the Turbulent Atmosphere on Wave Propagation, Israel Program for Scientific Translations, Jerusalem, 1971.

Wang, L. and Alexander, M. J.: Global estimates of gravity wave parameters from GPS radio occultation temperature data, J. Geophys. Res., 115, D21122, doi:10.1029/2010JD013860, 2010.

Wang, L., Geller, M. A., and Alexander, M. J.: Spatial and temporal variations of gravity wave parameters. Part I: intrinsic frequency, wavelength, and vertical propagation direction, J. Atmos. Sci., 62, 125-142, 2005. 